\title{
Logic programming: a tool for development of educational systems
}

\author{
Richard J. Allen \\ St. Olaf College \\ Northfield, Maryland \\ USA
}

\author{
Laurent Trilling \\ IMAG-LGI \\ Grenoble \\ France
}

\begin{abstract}
An intelligent system for exploring geometry is described. This system utilizes a declarative approach to geometric programming wherein geometric objects and properties are specified using logical specifications. The system automatically draws the specified figures from a set of designated base points and allows the user to explore geometric properties by dragging on these base points. Since the figure is constructed using a declarative approach, there are normally more points with which you can drag the figure than there would be if the figure had been constructed with a procedural system. The system has two parameters which the user must provide: the logical specification and the set of base points. The system, GéoSpécif is described. It is also shown how logic programming has been useful in defining and implementing a dynamic system for secondary school geometry and how a new programming construct, declarative geometric programming, grew out of efforts to design and build an educational system.
\end{abstract}

Main conference themes: artificial intelligence, software

Educational areas:

Study topics: mathematics

Secondary keywords: interactive, knowledge representation, learning systems, programming, tutoring 


\section{INTRODUCTION}

The aim of the GéoSpécif system is to automatically construct a geometric figure from a logical specification and a set of basic elements. The system provides a way of manipulating the figure by displacing one of the basics elements while respecting the logical specification. It is implemented entirely in Prolog III, a logic programming language with constraints. The use of constraints to implement this system has two merits: any linear construction can easily be carried out and many geometric constraints can be directly modelled using the built-in constraints of Prolog III. Moreover, we create a new paradigm - declarative geometric (logic) programming - the goal of which is to automatically construct a figure whatever the geometric constraints.

\section{INTELLIGENT TUTORS AND GEOMETRY}

Intelligent tutors (ITS) which are designed to help teach secondary students how to solve problems in geometry, make tools available:

- to aid in the construction of geometric figures which conform to a specification provided beforehand by a teacher;

- to stimulate discovery of important geometric properties represented in such figures;

- to guide the organization of proofs.

In previous work four main components of such systems have been identified [1]:

Figure acquisition: The student first constructs a figure which conforms to a specification provided by the teacher.

Figure appropriation: The student can graphically transform the figure while its logical properties are preserved.

Property exploration: The student reacts to system proposed interesting properties by using theorems furnished by the teacher.

Proof organization: The student, utilizing facts discovered during the previous steps and theorems furnished by the teacher, constructs a proof which is verified by the system.

\section{DECLARATIVE GEOMETRIC PROGRAMMING}

In this section we are primarily interested in the figure appropriation component which provides the capacity to transform a figure into as many different figures as possible; yet all of these figures respect the same underlying logical 
specification. Given a logical specification for a figure it can be constructed automatically from the objects and properties in the specification. And once constructed the figure can be transformed through displacement of any of its objects while the specification is still respected. For a student user this feature provides an invaluable tool for graphical exploration and discovery of properties induced by the logical specification.

Figure appropriation can be approached from two different perspectives: 'imperative geometric programming' and 'declarative geometric programming' [2]. The imperative geometric programming approach asks the student to produce a (procedural) construction of a figure which exhibits the order in which objects are to be constructed. Declarative geometric programming differs naturally from imperative programming in that a user is asked to provide only the logical specification of the figure. The system automatically constructs the figure, if possible, from whatever geometric objects are given. The figure can then be transformed by fixing all of the objects except the one which is used for doing the figure displacement. Transformed figures still respect the specification. This capability has significant implications for the design of teaching (didactic) situations which stimulate learning. Being able to animate a figure in all possible ways gives students the possibility of guessing more properties than if they were restricted to the animation induced by a particular order of construction found in an imperative geometric programming approach.

Constraint Logic Programming (CLP) [3] has proved to be an adequate Artificial Intelligence (AI) tool for supporting the declarative geometric programming found in GéoSpécif. We have used the following advantages of the CLP language Prolog III [4]:

- modularity expressed through rules permits easy extension or restriction of the system functionalities;

- calculus of constraints as exact solutions of linear constraints is provided by the language itself and resolution of the nonlinear constraints which we propose, can be expressed using this calculus;

- nondeterminism and suspended execution of goals (the freeze feature) permit solution of an important class of geometrical construction problems, namely those which can be solved by using linear calculus and relations expressed in logical specifications.

Our next goal is to obtain a better class where a notion of completeness is based only on geometrical criteria: construction can be done by using only objects which occur in the specification, and any properties deduced from the specification.

At this point it is important to note that systems such as Cabri-Géomètre [5, 6] and Sketchpad [7] have been the impetus for an exciting new concept: 
Dynamic Geometry. One could consider a 'dynamic figure' as an ordinary geometrical figure plus a certain means to move it while preserving its logical properties. These systems produce such figures and have proved to be very successful in helping students discover new properties in a figure. Essentially, these systems provide possible moves to the figures derived from a procedural construction given by the student. The system GéoSpécif represents a next step in the evolution of dynamic figures by providing more complete ways in which figures can be dynamic. GéoSpécif is able to guess a construction which has not yet been discovered by the student and the student can reasonably hope to have even more help than in the previously mentioned microworlds. For example, using the well-known locus method students could first construct a figure from any basic elements they want, then change elements in this figure to obtain the figure which conforms to the original construction problem. This can be done by weakening a condition on the object to be constructed and subsequently moving a basic object to observe the locus of the object to be constructed.

\section{GÉOSPÉCIF AND SYSTEMS OF EQUATIONS: LINEAR CASE}

Computer implemented Student Construction Languages (SCLs) [8] provide the basis for imperative geometric programming. Such languages provide an interface with which the users, typically school students, can:

- indicate on a menu what object (point, line, ray, segment, circle) they want to draw on the given graphics medium;

- draw the object;

- express the logical properties (name, belonging to another object, parallel to, perpendicular to, is the midpoint between, is the distance from, ...) of the drawn object with respect to already drawn objects.

The most important characteristic of the interface is that each operation must be realizable using drafting table instruments (ruler with translation, square, compass). Consequently users are limited in the constructions they can carry out. For example, students cannot construct a line passing through three points although they could create a line and afterwards three points belonging to it. Such an interface has been directly implemented in the system Mentoniezh [8] and similar interfaces have been implemented with the use of a mouse in the system Cabri-géomètre.

Our approach to declarative geometric programming is motivated in part by languages called Classroom Description Languages (CDLs) [8]. Such languages are inspired by those found in geometry textbooks. A specification for 
constructing a figure is a conjunction of atomic formulas composed of predicates. The specific ordering of the atomic formulas has no effect on the figure constructed. In the case of the intersection of the heights of a triangle $\mathrm{ABC}$ (Fig. 1), using our declarative geometric programming system, the figure can be constructed automatically from any three of the points $\mathrm{A}, \mathrm{B}, \mathrm{C}$ or $\mathrm{H}$ given the following specification:

point (A), point (B), point $(\mathrm{C})$, point $(\mathrm{H})$,

line (D4), line (D5), line (D6),

segment (S1, A, B), segment (S2, A, C), segment (S3, B, C),

$\mathrm{A} \in \mathrm{D} 4, \mathrm{~B} \in \mathrm{D} 5, \mathrm{C} \in \mathrm{D} 6, \mathrm{H} \in \mathrm{D} 4, \mathrm{H} \in \mathrm{D} 5, \mathrm{H} \in \mathrm{D} 5$,

$\mathrm{D} 4 \perp \mathrm{S} 3, \mathrm{D} 5 \perp \mathrm{S} 2, \mathrm{D} 6 \perp \mathrm{S} 1$

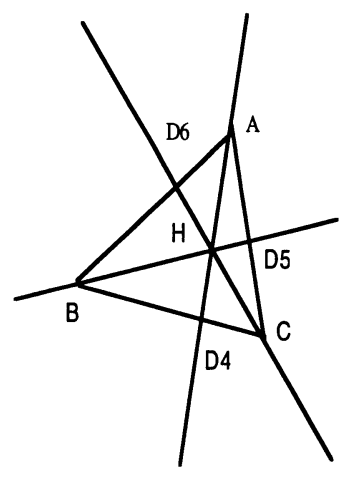

Fig. 1

A notable difference between this solution and an imperative one is that the point $\mathrm{H}$ can be relocated. One has to indicate what are base elements (for example A, B and $\mathrm{H}$ ) and which among the base elements is to be used for dragging (say $\mathrm{H}$ ). Our system then will automatically redraw the figure as we drag on $\mathrm{H}$ all the while respecting the specification. It is clear that such a system can readily be implemented in Prolog III since all equations derived from the above property specifications (with any three of the points $\mathrm{A}, \mathrm{B}, \mathrm{C}$ or $\mathrm{H}$ fixed) are linear. For $\mathrm{A} \in \mathrm{D} 4$ and $\mathrm{B} \in \mathrm{D} 5$, let $\mathrm{A}=(\mathrm{x}, \mathrm{y}), \mathrm{B}=\left(\mathrm{x}^{\prime}, \mathrm{y}^{\prime}\right)$ and let the lines D4 and D5 be defined by (slope, intercept) coefficient pairs $\left(a_{1}, b_{1}\right)$, $\left(a_{2}, b_{2}\right)$ respectively. 
Defining the belonging-to relationship algebraically we obtain the following two equations:
for $\mathrm{A} \in \mathrm{D} 4$
(1) $y-a_{1} x-b_{1}=0$
for $\mathrm{B} \in \mathrm{D} 5$
(2) $y^{\prime}-a_{2} x^{\prime}-b_{2}=0$
with $a_{1}, b_{1}, a_{2}, b_{2}$ given.

There are four unknowns $x, y, x^{\prime}, y^{\prime}$. On the one hand equations (1) and (2) provide a linear relation between $y$ and $x$ and a linear relation between $x^{\prime}$ and $y^{\prime}$ on the other. These two equations can be solved for $\mathrm{x}, \mathrm{y}, \mathrm{x}^{\prime}$ and $\mathrm{y}^{\prime}$ under certain conditions which can be expressed algebraically, and also in an equivalent geometric form. The exploitation of these conditions in the context of a dynamic system provides opportunities to effect cross-fertilization between linear algebra and geometry. In Prolog III any calculations carried out on the equations are exact. This means-and this is extremely important-that (linear) overconstrained specifications can be accepted. Overconstraints are those which are not necessary for the construction of the figure, but which must nevertheless be verified. Such specifications arise very naturally. For example the preceding specification where $\mathrm{A}, \mathrm{B}$ and $\mathrm{C}$ are fixed, belongs to this category; the fact that $\mathrm{H}$ is the intersection of three lines has to be verified.

\section{Nonlinear case}

Problems become nonlinear if we add formulas of the type $|A B|=d$ to our specification. The solution of systems of such equations, namely equations of lines $(y=a x+b)$ and equations of circles $\left(\left(x-x^{\prime}\right)^{2}+\left(y-y^{\prime}\right)^{2}-d^{2}=0\right)$ together, is discussed here in the context of the design and implementation of GéoSpécif. Their satisfiability is known to be decidable. It is also known that a construction using ruler and compass can be found if it exists (whereas in the general case construction has to be carried out numerically).

An example of a nontrivial problem (typically asked of 13-14 year olds in French schools) follows:

Suppose that the lines $\mathrm{D}, \mathrm{D} 1$ and $\mathrm{D} 2$ along with the distance $\mathrm{d}$ are given. Construct points $A$ and $B$ such that $|A B|=d, A \in D 1, B \in D 2, A B / / D$ (Fig. 2). Let $A=(x, y), B=\left(x^{\prime}, y^{\prime}\right)$ and let the lines $D 1, D 2, A B$ and $D$ be defined by (slope, intercept) coefficient pairs $\left(a_{1}, b_{1}\right),\left(a_{2}, b_{2}\right),(a, b)$ and $\left(a^{\prime}, b^{\prime}\right)$ respectively. 


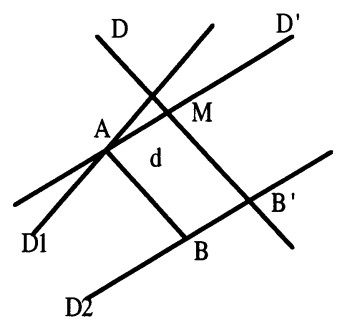

Fig. 2

From the properties given we obtain the system of equations:

$\begin{array}{ll}\text { from }|A B|=d & (1)\left(x-x^{\prime}\right)^{2}+\left(y-y^{\prime}\right)^{2}-d^{2}=0 \\ \text { from } A \in D 1 & (2) y-a_{1} x-b_{1}=0 \\ \text { from } B \in D 2 & (3) y^{\prime}-a_{2} x^{\prime}-b_{2}=0 \\ \text { from } A B & (4) y-a x-b=0 \\ \text { from } A B & (5) y^{\prime}-a x^{\prime}-b=0 \\ \text { from } A B / / D & \text { (6) } a=a^{\prime} \\ \text { with } d, a_{1}, b_{1}, a_{2}, b_{2}, a^{\prime} \text { and } b^{\prime} \text { given. }\end{array}$

There are six unknowns $\mathrm{x}, \mathrm{y}, \mathrm{x}^{\prime}, \mathrm{y}^{\prime}, \mathrm{a}$ and $\mathrm{b}$. On the one hand equations (2) and (3) provide a linear relation between $\mathrm{y}$ and $\mathrm{x}$, and a linear relation between $\mathrm{x}^{\prime}$ and $y^{\prime}$ on the other. Similarly equations (4) and (5) provide other linear relations between $x$ and $y$, and between $x^{\prime}$ and $y^{\prime}$. These four equations together with equation (6) allow us to solve for $y, x^{\prime}$ and $y^{\prime}$ as linear functions of $x$. This in turn allows equation (1) to be solved for $x$. It is interesting to note that an imperative solution to this problem using compass and ruler requires introduction of a new line: Let $B^{\prime} \in D 2, B^{\prime} \in D$ and $M$ such that $\left|B^{\prime} M\right|=d$. Then introduce the line $\mathrm{D}^{\prime} / / \mathrm{D} 2$ with $\mathrm{M} \in \mathrm{D}^{\prime}$ and we obtain $\mathrm{A} \in \mathrm{D}$ 1.

Some problems are not solvable using our method, but would be if a little more geometric information were provided in the specification. For example, to construct a tangent from a given point $P$ to a given circle of centre $O$ and radius $\mathrm{r}$, the specification is expressed as follows: Given points $\mathrm{O}$ and $\mathrm{P}$ and distance $r,|\mathrm{OM}|=\mathrm{r}$ and $\mathrm{OM} \perp \mathrm{PM}$ (Fig. 3). The reason our system is unable to do the construction is that it does not know that $\mathrm{M}$ belongs to the circle whose diameter is OP. Educationally we can exploit these situations by asking the student to suggest what might be added to the specification so as to create a solution; the student has an interactive tool in our system with which to try out conjectures. 
This last example suggests a simple way of overcoming the lack of geometric information available to our system: ask the user's help in providing it. Since the user is supposedly working with the system to obtain more flexibility in animating figures, this suggests a willingness to help the system achieve better results.

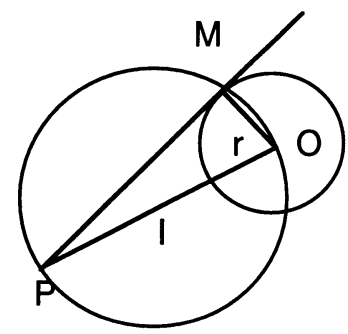

Fig. 3

More exactly we are asking the user to provide the system with overspecifications. If we add the overspecifications: $\mathrm{I}=$ midpoint $(\mathrm{O}, \mathrm{P})$ and $|\mathrm{OI}|=|\mathrm{IM}|$ in our last example, then $\mathrm{M}$ can be constructed as the intersection of two circles. Such overspecifications are of course translated into overconstraints; this leads us to the issue of solving overconstraints in nonlinear cases. It is clear that teachers can utilize our system for student exploration and verification of conjectures concerning overspecifications and their geometric significance.

\section{Overconstraints}

Overconstraints arise naturally. Not only do these provide means whereby users can interact intelligently to help the system, but these also can be very naturally and unconsciously introduced into a specification as in the case of the intersection of the heights of a triangle. Thirdly, these might be introduced automatically by our system as it creates new lines (common chords of intersecting circles) when needed. Attempts to address the overconstraint issue raise many mathematical, educational and technological issues. The calculations in the linear case are exact (Prolog III), whereas if the solutions to two quadratic equations are known to be the same algebraically, we cannot be assured of always computing these equal numerically. The problem of solving overconstraints in general requires symbolic computation. This contrast between the mathematics and the limitations of the technology is a striking dilemma. 


\section{CONCLUSION}

Designing educational systems appears to pose concrete problems which constitute a challenge for logic programming. The following avenues, already under development, appear promising:

- logic programming languages offering extended Horn clauses for treating classical negation and abductive reasoning [9];

- Inductive Logic Programming for identifying a student learning model which provides appropriate explanations [10];

- algorithms for nonlinear constraints which are complete with respect to a semantics expressed in geometric terms. As mentioned before such a completeness is required for educational purposes. We also hope to obtain good results in this case by using constraints on intervals [11] (the general case is known to be very difficult to solve [12]).

\section{REFERENCES}

1. Allen, R., Nicolas, P. and Trilling, L. (1990) Figure Correctness in an Expert System for Teaching Geometry, in Proceedings of the eight biennial conference of the Canadian society for computational studies of intelligence, Ottawa, Canada, pp. 154-160.

2. Allen, R., Idt, J. and Trilling, L. (1993) Constraint Based Automatic Construction and Manipulation of Geometric Figures, in Proceedings of the 13th International Joint Conference on Artificial Intelligence, Chambéry, France, pp. 453-458.

3. Cohen, J. (1990) Constraint Logic Programming Languages. Communications of the ACM, 33 (69).

4. Colmerauer, A. (1990) Prolog III. Communications of the ACM, 33 (69).

5. Baulac, Y., Bellemain, F. and Laborde, J.M. (1988) Cabri-géomètre, Manuel d'utilisation. Cédic-Nathan, Paris.

6. CABRI Geometry II (1994) Texas Instruments, Dallas, Texas.

7. Sketchpad (1994) Key Curriculum Press, Berkeley, California. 
8. Nicolas, P. (1989) Construction et vérification de figures géométrique dans le système Mentoniezh. Thèse de l'Université de Rennes I.

9. Loveland, D.W. (1897) Near-Horn Prolog. in Proceedings of the Fourth International Conference on Logic Programming. Lassez, J.-L. (ed.). Melbourne, Australia, MIT Press.

10. Muggleton, S. (1992) Inductive Logic Programming. The Apic Series No. 38, Academic Press.

11. Older, W. and Vellino, A. (1994) Constraint Arithmetic on Real Intervals, in Constraint Logic Programming: Selected Research, Colmerauer, A. and Benhamou, F. (eds.). MIT Press.

12. Chou, S.C. (1988) Mechanical Geometry Theorem Proving. Reidel Publishing, Norwell, MA. 